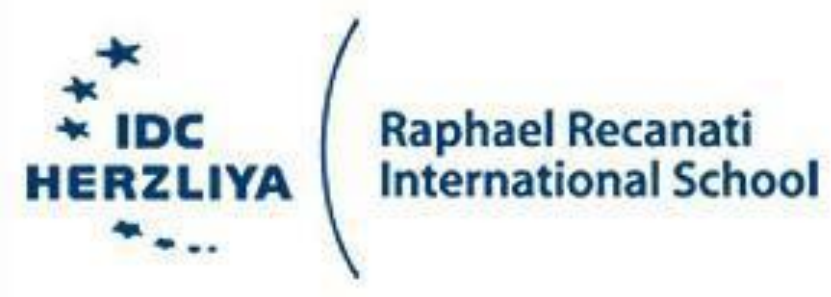

Seminar Paper

Advanced Topics in Social Network Analysis

\title{
A Qualitative Analysis of a Team Fortress 2 Social Network
}

\author{
Submitted by: \\ Ilan Tollman, Motty Yaffe
}

Date: 01/10/2015 
Table of Contents

- Introduction 3

- Background 4

- Related Work 6

$\begin{array}{ll}\text { - Theory } & 6\end{array}$

$\begin{array}{ll}\text { - Prior Research } & 7\end{array}$

- Methodology 11

$\begin{array}{ll}\text { - Results } & 13\end{array}$

$\begin{array}{ll}\text {-Discussion } & 15\end{array}$

$\begin{array}{ll}\text { - Conclusion } & 17\end{array}$

$\begin{array}{ll}\text { - References } & 18\end{array}$

$\begin{array}{ll}\text { - Appendix } & 19\end{array}$ 


\section{Introduction}

The Gaming Industry is one of the most dynamic and fasted growing of our time, from the arcade culture to the widespread integration of household gaming consoles and home gaming PCs, whose steadily rising popularity, performance and inter-connectivity is allowing for more and more growth in the range and permeability of gaming and gaming culture. With its widespread popularity and its ever-increasing influence, through the acceptance of gaming culture as mainstream, the gaming world is becoming a crucial field of study for better understanding of online communities and social interaction. In this paper we will expand on the academic field of Online Social Network Analysis through the examination of a selected social network from the gaming world as a microcosm of online social interactions. The paper will draw on Social Network Analysis, Sociology and Communications theories to further expand the understanding of on-line interactions on a network scale.

Current gaming focused research leads in two main directions, social consequence whether they be pro social or anti social. There is also however a smaller developing trend of study within the Social Network Analysis field that uses the data collected by computer game makers to study network structure as a variable of its own. In this research the findings of this trend within the computer game research will be used to conduct a qualitative Analysis of a small friends list network from Team Fortress 2. The data collected will compared and contrasted with established Sociology and Communications theories, the expected results and variances will then be explained using the findings of Social Network Analysis research.

The Research draws the Two Step Flow communications theory (Lazarsfeld, 1948), and a slightly more refined version of the theory (Katz, 1957), to deduce relevant information on the interaction between companies, hardcore gamers and more casual gamers. The study will also draw predominantly on Mark Granovette work on Social Network Analysis. 


\section{Background}

The game being studied in this paper is Team Fortress 2, it is an iconic part of the one biggest and most diverse gaming corporations, Valve Inc. The Company set itself apart from its inception, it started out by releasing half life, an arcade style first person shooter than ran on the Doom engine (Dunn, 2013), the distinction between it and the many similar games of the time, were that valve let the game have a long shelf life, keeping it as their main game for about six and a half years. Their next move involved purchasing Team Fortress, the prequel to the game studied in this paper. Despite the fact that both games were already released, the company took an unusual route for the early nineties and two thousands encouraging the enthusiastic modding communities by releasing SDK (software developments kits) for free. Modders, are gamers/developers who modify games with the intention of creating new content and game play.

The most successful mod to come from this new type of development was Counter-Strike, the creators of which were hired by Valve and were able to successfully release one of the biggest games of the era. This new consumer interaction which enlisted the company's audience into their development proved hugely profitable and while many other companies have now encouraged modding communities, none have been able to market it as well as Valve. This concept of utilising communities in order to create content has become a staple of internet culture, but few have seen the profits the gaming world has.

An interesting example of the success of modding is exemplified by looking back to Warcraft, a Blizzard real time strategy game which spawned not only an entourage of hugely popular mods but also a handful of brand new gaming genre's. The game involved commanding an army, hero, and production city simultaneously while trying to wipe out your opponents. The high multi tasking demand put on the player led Blizzard to create a few simpler variations of the game, Starcraft, wherein the hero no longer existed, and World of Warcraft where the hero was the only character controlled by the player. Many of the fans of the original game continued playing it however and modders continued to create new gameplay. The most influential of these mods was DOTA or Defence of the Ancients developed and maintained by Icefrog, who's notoriety is in part due to his 
Anonymity. DOTA marked the beginning of the MOBA, multiplayer online battle arena game genre, which is currently the forerunner in popularity amongst the competitive e-sports gaming world. After approaching Blizzard with the intention of developing the Mod into a fully fledged game and being rejected, Icefrog joined up with Valve and began working on a sequel to the original mod. At the same time a separate gaming company, Riot Gaming, developed the next big game in the new MOBA genre, League of Legends. League of Legends and DOTA2 are currently two of the biggest games competitive e-sports with annual competitions sporting millions in prize money and millions of dedicated fans.

Team Fortress 2 is the sequel of the 1996 Team Fortress mod for the game Quake developed by id software. The incorporation and utilization of mods, even when developed from other companies games seems to be a core value in Valve's business model, a kind of be all that you can be for computer games. Considering this, Valve was chosen as the appropriate company for this study, and Team Fortress 2 as relatively new and still popular game with readily available data on the community was a prime candidate for studying network structure as a variable in gaming communities. 


\section{Related Work}

\section{Theory}

The Research draws the Two Step Flow communications theory (Lazarsfeld, 1948), and a refined version of the theory (Katz, 1957), to deduce relevant information on the interaction between companies, hardcore gamers and more casual gamers. The theory is a political communications theory based on a research of voting behaviour which examined and questioned voters regarding where they got the information they used to base their voting decisions. This research assumes a similar information flow in other fields. The research found that the information flow could be modelled as such that official sources would be tuned into by a select knowledgeable few known as opinion leaders, who then distil the information and communicate relevant opinions to their friends family and acquaintances. In this way the opinion leaders were the ones to critically evaluate official sources, and frame the relevant information accordingly. This research assumes the computer games and game manufacturers as official sources or media outlets, opinion leaders as hardcore gamers, modders and game reviewers (such as youtubers) and the voting public as the casual or bulk of regular gamers.

The study will also draw predominantly on Mark Granovette work on Social Network Analysis. Granovette is one of biggest academic contributors to the field of social network analysis and his work on the strength of weak ties (Granovette, 1973) and embeddedness (Granovette,1985). Similarly the Social Network Analysis concept of the Tipping Point (Gladwell, 2000) will be used in conjunction with the aforementioned theories to compliment the framework within which this paper is written. These theories and concepts are further developed on the related works section of this paper. 


\section{Prior Research}

While a significant amount of research has been conducted at the intersecting fields of computer games and Social Network Analysis, the questions most of them seem to address are the significance of Social Network Analysis as a variable? and how serious of a subject are video games in realm of social sciences? There is also the more commercial side of research in general that attempts to answer the questions of information flow and eWOM, electronic word of mouth (Chen et al, 2014; Hayat, Brainin \& Neter, Forthcoming) . This paper deviates in these fields by combining the robust studies measuring the validity of Social Network Analysis measures such as on Virtual Brokerage and Closure (e.g., by Hayat \& Mo, 2015; Shen et al, 2012; Mo et al, 2015), Social capital, selfesteem, and use of online social network sites (Steinfield et al, 2009; Hayat, Lesser \& Samuel-Azran, Forthcoming) and more widely applicable research such as Granovette's work and Lazersfelds two step flow theory (1948).

Granovette's book the strength of week ties (1973) answered a major question regarding the application of Social Network Analysis, by being able to empirically show the significance of network structure on something as basic as finding a job, he was able to highlight the significance of this field of study. His research analysed the impact of network structure on job searching, the findings showed that people in networks with many weak ties were much more successful job hunting than those with mainly strong ties. What this means is that certain network structures are contextually advantageous, and these network structures have measurable variables.

In his work on embeddedness (Granovette, 1985), the significance of Network structures is extended beyond just being a contributing variable, and an argument is made that it is in fact an inextricable variable, one wherein the context of economics must always be considered. While this argument was made in the contexts of economics, the implications extend to all social roles, the impact of relationships (e.g., Hayat \& Lyons, 2010), culture, moral, culture and politics (e.g., Hayat, Samuel-Azran \& Galily, 2016) are all contributing factors to network structure which in turn impact the individuals within them. 
Shen et al's (2014) work on video games was able to empirically show both the advantageous nature of video games as an eco system for network structure research and to further substantiate the quantifiable aspects of network structures and their relation to individuals. The study focused on Social Capital when defined as the value the individual can get from the social relations. In the context of an MMORP this value was given as success and enjoyment within the game. The social relations were defined in network terms as Brokerage and Closure.

Brokerage as a term relates to concept previously introduced in Granovette's (1973) the strength of week ties, instead of defining term as individuals to whom the subject has week ties, acquaintances or simply distant friends, because of the amount data that is available in such games the study defined it more concretely as brokers, people with a greater diffusion of ties, meaning players who's friends are not friends with each other. This creates a more diverse network wherein players are not exposed to redundant information, skills, resources and strategies. As such players known as Brokers with a high brokerage score were expected and then later shown to progress much more quickly in game and gain more achievements. On the flip side Closure as related to Granovette's strong ties, refers to players in dense networks were all the friends are friends with each other. As was expected the converse was expected for these players, what was also shown however was that these players generally trusted their friends more. This measure of trust as predicted by the closure of players is introduced as bonding social capital.

What this study was able to introduce with regards to the significance of network structure, is that player's overall experience can be predicted using social network structure variables, if one was to assume a causational relationship it would also be reasonable to deduce that by controlling for these variables it would be possible to shape these aspects of the persons game play. The replication of Granovette's work in this research using its robust controls and the surplus of available data and resulting in the same findings also suggests that research conducted within games can to an extent be generalized to none-game social settings. 
With regards to the current research this paper supports Shen et al's use of Social Network measures and variables as predictors for game play, allowing for a better understanding of each of the individual players examined using their location within the network. Additionally it supports the concept that the findings which are made possible through the computer game platform can be contextually generalized to other none game related networks, such work place, academia and other online communities.

Additional work in the field of Social network analysis serves to tie together the social network variables commonly used to examine individuals with more commonly studied psychological phenomenon. Social capital, self-esteem, and use of online social network sites (Steinfield et al, 2008) used Facebook as a platform to examine the psychological well being of students. The research contrasted Facebook usage with psychological well being of students, utilising Facebook usage as the independent variable wherein the time students spend on Facebook was measured, this was then expected to have an impact on the students psychological well being, the dependent variable. The interesting aspect of the study as it relates to this work, was the usage of network measures as contingent variables, used to emphasize and explain the main subjects being studied more so than to focus on them individually. In this manner the research was able to show the indirect link between the two variables being studied by analysing the bridging social capital afforded to participants who used Facebook heavily in large heterogeneous social networks. The use an additional research field which in this case was Social Network Analysis, also enabled the research to better explain the context necessary for Facebook to have this positive effect. It was made possible to show that it was users who suffered with low self esteem who gained most from the use of Facebook, wherein the affordances that Facebook offers, which is mediated interaction served the more shy students more so than the rest. The significance of this research is that it further expands the utility of the field of Social Network Analysis, whose measures are often overlooked due to the indirect nature of their interaction. Instead of focusing on cause and effect phenomenon it is also possible to use Social Network Analysis in more in depth research in order to explain those phenomenon 
Tipping Point by Malcolm Gladwell (2000), takes a none-network approach to understanding fads, crowds and information flow, as opposed to using the network structure to predict information flow he uses individuals from within the network, connectors, mavens and sales men. When relating these to the Social Network field it is easy to see the relations, connectors would be very central nodes within the network, mavens would be the specialists or experts and sales men would be the charismatic such as the PR department of marketers. These concepts can however also be applied to gamers wherein the social gamers would be the connectors, the hardcore gamers would be the mavens and salesmen would simply be those who come up with most catchy slogan as develops naturally with player's nature. In MMORGs some of these ideas were explored with the introduction of allowing guilds to be formed within the gaming platform and each players expertise within the guild determined based on rank within in the guild or even avatar level.

Combining all the aforementioned theories and concepts developed in the various fields with their complimenting and interlocking ideas, it becomes clear that these studies on social interaction, economic nature, psychological implications, and network analysis can be used to garner and develop rather significant insights into both individuals within networks and the networks themselves and accordingly make significant predictions with regards to how various manipulations may affect the participants. What this study proposes is that within the gaming eco-system, the readily available data, clear and definitive goals and its similarity to external social networks allows for extensive research with more controls to help further understand Social Networks. Accordingly this research suggests that combining all the previously mentioned studies, can be used to better understand the information which can be collected from a sample network within this eco system? The hypothesis therefore is that through analysis of the network structure it is possible to make predictions regarding both individuals and the network itself within the gaming eco system using theories developed in none gaming social scenarios. 


\section{Methodology}

The research conducted was both qualitative and descriptive, the researches gathered a sample from the prescribed population with the intention of comparing the available data and its subsequent analysis with previously validated theories. In this way the study supports the video games platform as a research methodology which can be generalized to the general public.

The population being studied in this paper is computer gamers on online platforms where social interaction is encouraged through chat options, friends lists, guilds etc. the gamers in particular also have to be self contained within the platform as an eco system, in this study the platform chosen was Steam which offers friends lists, chat options, in and out of game trading, groups, developer starter kits to allow for player created content, a platform for sharing player created content, avatar cosmetics which once again can be traded. The overall population however combines that of this study and referenced studies to provide additional external validity to future computer game based social network research.

The sample was chosen from the game Team Fortress, one of Valves iconic games which is both freely available on Steam and also fully integrated into the Steam platform. The game is a product of Valve's initiative to involve its fan communities in its development of software, which highlights the companies view of the value of understanding and interacting with game development company's communities. The game is also expected to incorporate a new MMR match maker rating system which will introduce a new competitive aspect to the game, which may allow it to take a similar route to Counter Strike or Dota 2, if this does take place the data available in this research may be useful for future researcher to analyse how the introduction of a new competitive aspect affects network structure. The actual sample was chosen by choosing a player at random, whichever was first encountered by the researchers and who's profile was set to public, this player friends list was then requested through the API and the friends lists of all his friends was also created and as such creating a two degree's of separation ego network. 
The Data was collected using the openly available Steam API, a dedicated server which one can sign up for by becoming a Steam developer which is once again freely accessible. The server sends automated responses with the requested data, because the sample size in this research was relatively small the requests were processed manually and the data inputted manually. For larger scale studies, programmes can be written to collect data extremely quickly and without too much manpower. The initial player, alias participant 0 had a friends list of only six other players, once those six players friends lists were pulled a total of ninety three participants were chosen and their information requested through the API and collected. Additional information was requested and collected by the researchers according to its relevance and availability.

The collected data was and then input, rather appropriately, into a modded version of Excel which was altered to specialize in Social Network Analysis NodeXL. In this programme a number of Analysis were conducted to produce a graph showing each participant as a node and their friendship as a connection between each node. This graph gave an overall overview of the network as a whole. The data was then run through a series calculations to get Social network analysis measures for the entire network and individuals in the network such as the various centrality measures. An additional important note to make is that despite the large amount of accessible data the sparse nature of the network meant that an accurate clustering co-efficient was not calculated. 


\section{Results}

The results of mapping the various connections in NodeXL created a graph seen below, it is clear from the graph that there are few very central individuals with a high degree centrality. There are also a small number extra nodes with a low but existent degree centrality of greater than two but less than five. It is clear that by Social Network Analysis standards this network would not be defined as a community (Wellman et al., 2012) with a clustering co-efficient of 0.4 or higher. The clustering co-efficient is the measure of the number of connections within a network divided by the number of possible connections within a network and can be used to attest to how well connected the community is.

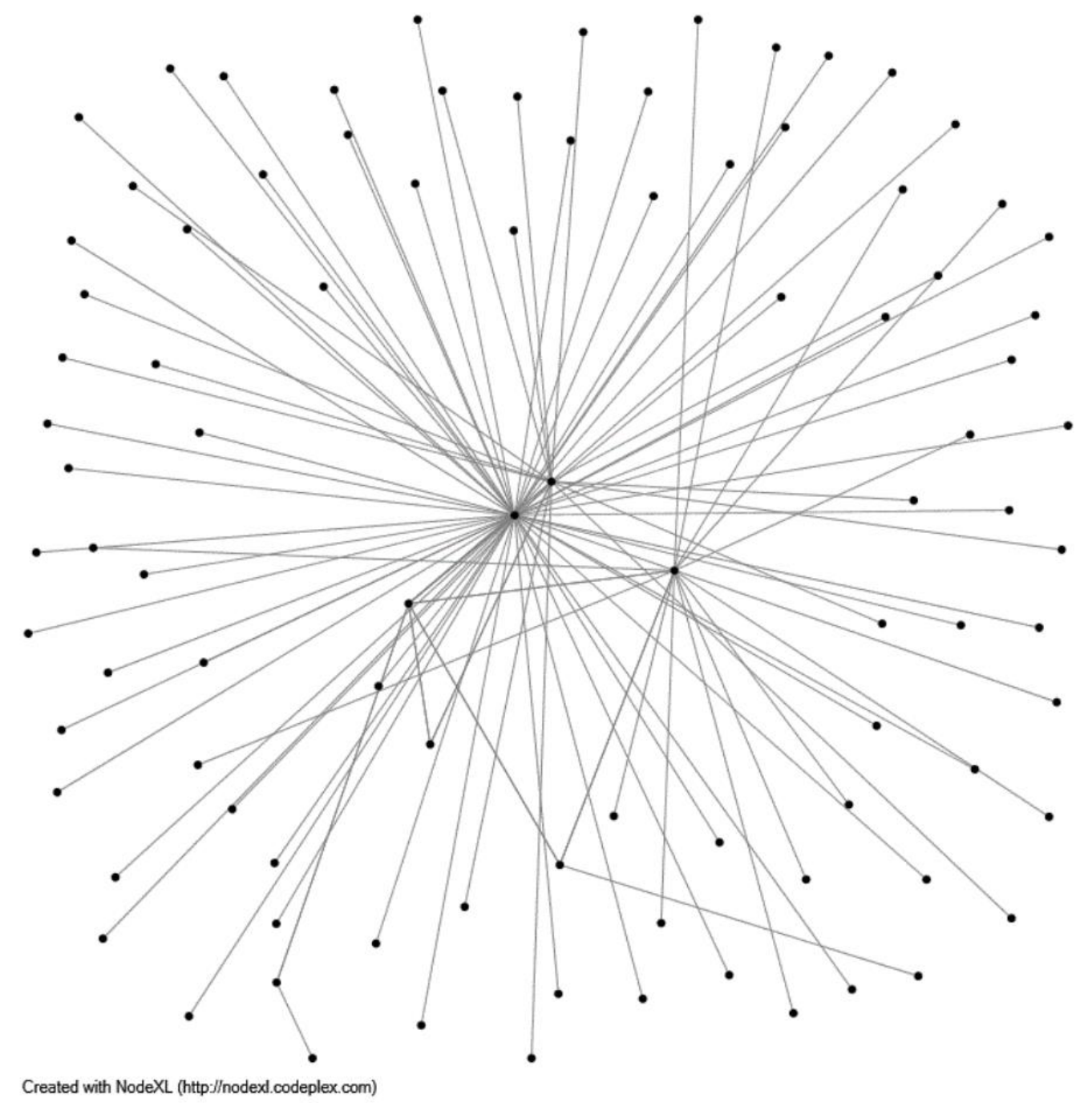


Participant zero, or the initial user upon which this network was built only has a degree centrality of six of but still features strongly toward the centre of the graph, this would be expected due the nature of how the sample was collected, because this is a two degrees of separation ego network it is expected that he would show prominently even when his degree centrality is relatively low. The results illustrate participants zero's role in the construction of the network despite his low degree centrality by scoring him second highest on betweeness centrality, the measure through which each node is as closely connected to every other node through him. He also scored highest his Closeness centrality measure alongside the participant with the highest degree centrality alias Mr. popular. Participant zero scored second highest by a small margin on his Eigenvector centrality measure which takes into account the most factors in calculating centrality, this of course would be as expected, since the Eigenvector centrality measures the importance of a node within a network and Participant zero was the node around which the network was constructed. Game play for Participant zero, he had the twenty ninth most in game achievements out of eighty three (64), he had the twenty first most items in game out of thirty three (45), and he has the twenty second most time played out eighty three (6256).

Mr Popular, the participant with the highest degree centrality, had the highest of all the centrality measures, considering his direct connection to over half the network this seems obvious. Mr Popular had the twenty second most in game achievements out of eighty three (113), he also had the twelfth most time played out of eighty three (14024) in the network.

To compare the relative success of the major nodes within the Network the average number of achievements earned by players was eighty three, the average number of player items was one hundred and twenty six and the average time played was seven thousand five hundred and eighty seven minutes. Considering these figures both of our most central nodes under preformed, when considering alongside that the player with the most achievements alias Super Gamer with two thousand and thirty six minutes played and five hundred and eighteen achievements it becomes evident that central nodes game time to performance ratio is sub-par. 


\section{Discussion}

The first insight offered by the results suggests that the gaming eco system, similar to outside eco systems are context aware, the results were a bit surprising in that they gave an insight into the type of game that Team Fortress 2 is. The game consists of repeating rounds of territorial control, capture the flag and control point, after each round the game is completely reset, teams are shuffled and you start again, the characters are comedic caricatures and the overall feel of the game is that of a fun loving shoot em up. With this in mind the network structure took the shape of a sparse, high degree but low density network similar to what you would expect of something like a party or night club. Accordingly the more social gamers such as Participant zero and Mr popular ended up under performing because of what can be assumed as their goal in the game being more so for the sake chilling with mates as opposed to Super Gamer who got the most achievements with less than a fifth of their time played. Therefore this research would argue that this network structure is more suited for a fun and games approach but in general will lead to under productivity.

The second insight offered by this research see's the under utilisation of information flow and the Mavens/Opinion leaders (Gladwell, 2000), (Lazarsfeld, 1948), they do not achieve important positions within the network due to the lack of competitiveness or seriousness. A potentional prediction for future research, once the MMR system is introduced to Team Fortress 2, would be that power players such Super Gamer would take more central role within the network and that Social gamers such as Participant Zero and Mr Popular's network position will become secondary on the other hand their position within the network as it is currently will allow them to utilize the opinion leaders/mavens and as such their performance would improve. To incorporate aspects of Shen et al's (2012) research on social capital, brokerage and closure, one would expect the introduction of a more cluster and bridge orientated network, or a more robust once more permanent incentives such as MMR are introduced. Comparatively, the current network structure gives the impression of being purely social, and lacking in the additional motivations shown in their research such as trusted clusters and bridging mentors. 
Unfortunately, the available data was not sufficient to fully answer the research question, but rather it was just able provide some support toward the insight afforded by examining network structure. As such, the hypothesis is supported, and offers to further strengthen previous studies on the value of network structure and network variables on understanding communities. The important answer provided by the data as viewed by the researchers is that network structure is heavily influenced by the motivations of communities. If these motivations are temporary and fleeting as in Team Fortress 2 the network structure ends up being sparse and to a certain extent random. If the motivations are more structured and competitive as is seen in Granovette's (1975) work on job searching or even Shen et al's (2012) on MMORPG games the network structure becomes more concrete and begins to follow patterns the relate more strongly to research conducted by Gladwell (2000) and Lazarsfeld (1945) with important individuals occupying central positions within the network.

I think the insights introduced in this study would be valuable to future research as mentioned previously in reconstructing this research once the competitive aspect has been introduced and examining the subsequent effect to the network structure. As such one limitation of this research of as a result of lack of time not being able to continue this study in a longitudinal fashion and compare and contrast the network structure over time. Additionally and in the same line of thought, it may be of value to re-examine traditional online social networks such as Instagramme and Facebook wherein the scoreboard is removed (number of likes, followers, shares, etc.) and see if the opposite may also be true, that by removing a competitive aspect the network structure would be compromised and begin to follow a more random and sparse structure. 


\section{Conclusion}

Gaming platforms not only offer an efficient and robust eco-system within which to study network structure but also a variety of network types which can be related to different types of networks. The industry itself is one of the biggest in the world, and has thus far made extensive use of its own networks, communities and fans. This research is just one example of the usefulness of both Social Network Analysis as a field of research and Computer Gaming as a platform through which to utilize big data. While this research failed to implement the theoretical framework to the network, it was able to show the limitations of that theoretical framework and the importance of particular network structures to being able to successfully implement particular theories. Additionally this research was able to show that despite this limitation important and relevant information can still be garnered from an in depth qualitative analysis of the Network structure within just a simple understanding of the communities context. The hypothesis in this research was supported and a partial answer was provided for the broad research question. 


\section{References}

Chen, Y., Tang, K., Wu, C., \& Jheng, R. (2014). Predicting the influence of users' posted information for eWOM advertising in social networks. Electronic Commerce Research and Applications, 13(6), 431-439.

Dunn, J. (2013, October 4). Full Steam ahead: The History of Valve. Retrieved from http://www.gamesradar.com/history-of-valve/

Gladwell, M. (2000). The tipping point: How little things can make a big difference. Boston: Little, Brown.

Granovetter, M. (1975). Strength of Weak Ties. Encyclopedia of Social Theory.

Granovetter, M. (1985). Economic Action and Social Structure: The Problem of Embeddedness. The American Journal of Sociology, 91(3), 487-487.

Hayat, T. Z., Brainin, E., \& Neter, E. (Forthcoming). With some help from my network: supplementing eHealth literacy with social ties. Journal of medical Internet research, 19(3).

Hayat, T. Z., Lesser, O., \& Samuel-Azran, T. (Forthcoming). Gendered discourse patterns on online social networks: A social network analysis perspective. Computers in Human Behavior, 77, 132-139.

Hayat, Z., \& Lyons, K. (2010). The Evolution of the CASCON Community: A Social Network Analysis. CASCON $20^{\text {th }}$ Annual International Conference. Toronto, ON, Canada.

Hayat, Z., \& Mo, G. Y. (2015). Advice giving and receiving within research networks. American Behavioral Scientist, 59 (5), 582-598.

Hayat, T., Samuel-Azran, T., \& Galily, Y. (2016). Al-Jazeera Sport's US Twitter followers: Sport-politics nexus?. Online Information Review, 40(6), 785-797.

Lazarsfeld, P., Berelson, B., \& Gaudet, H. (1984). Erie County Study, 1940. ICPSR Data Holdings.

Mo, G. Y., Hayat, Z, Wellman, B. (2015). How Far Can Scholarly Networks Go? Examining the Relationships between Distance, Disciplines, Motivations, and Clusters. Studies in Media and Communications, 9, 107 133.

Shen, C., Monge, P., Williams, D. (2014). Virtual Brokerage and Closure: Network Structure and Social Capital in a Massively Multiplayer Online Game Communication Research, 41 (4), pp. 459-480.

Steinfield, C., Ellison, N., \& Lampe, C. (2008). Social capital, self-esteem, and use of online social network sites: A longitudinal analysis. Journal of Applied Developmental Psychology, 434-445.

Wellman, B., Rainie, L., Hayat, Z., \& Beermann, C. (2012). The Future of Networked Individualism. In Lee R. \& Barry W. (Eds), Networked: The New Social Operating System. (pp. 275-303). Massachusetts: MIT Press. 


\section{Appendix}

\section{Vertex 1}

Vertex 2

76561198149180078

7656119

76561198149180078

7656119

76561198149180078

7656119

76561198149180078

7656119

76561198149180078

7656119

76561198149180078

7656119

76561198129085144

7656119

76561198129085144

7656119

76561198132684906

7656119

76561198132684906

7656119

76561198132684906

7656119

76561198132684906

7656119

76561198132684906

7656119

76561198132684906

7656119

76561198132684906

7656119

76561198132684906

7656119

76561198132684906

7656119

76561198132684906

7656119

76561198132684906

7656119

76561198132684906

7656119

76561198132684906

7656119

76561198132684906

7656119

76561198132684906

7656119

76561198148113785

7656119

76561198148113785

7656119

76561198149237454 
76561198149237454

7656119

76561198149237454

7656119

76561198149237454

7656119

76561198149237454

7656119

76561198149237454

7656119

76561198149237454

7656119

76561198149237454

7656119

76561198149237454

7656119

76561198149237454

7656119

76561198149237454

7656119

76561198149237454

7656119

76561198149237454

7656119

76561198149237454

7656119

76561198149237454

7656119

76561198149237454

7656119

76561198149237454

7656119

76561198149237454

7656119

76561198149237454

7656119

76561198152532873

7656119

76561198152532873

7656119

76561198152532873

7656119

7656119

7656119

7656119

7656119

7656119

7656119

7656119

7656119

7656119

7656119

7656119

7656119

7656119

7656119

7656119

7656119

7656119

7656119

7656119

7656119

7656119

7656119

7656119

7656119

7656119

76561198152532873

7656119

76561198152532873

7656119 
A Qualitative Analysis of a Team Fortress 2 Social Network

76561198152532873

7656119

76561198152532873

7656119

76561198152532873

7656119

76561198152532873

7656119

76561198152532873

7656119

76561198152532873

7656119

76561198152532873

7656119

76561198152532873

7656119

76561198152532873

7656119

76561198152532873

7656119

76561198152532873

7656119

76561198152532873

7656119

76561198152532873

7656119

76561198152532873

7656119

76561198152532873

7656119

76561198152532873

7656119

76561198152532873

7656119

76561198152532873

7656119

76561198152532873

7656119

76561198152532873

7656119

76561198152532873

7656119

76561198152532873

7656119

76561198152532873

7656119

76561198152532873

7656119

76561198152532873

7656119

76561198239562328

7656119

76561198239562328

7656119

76561198239562328

7656119 
76561198129085144 76561198132684906 76561198148113785 76561198149237454 76561198152532873 76561198239562328 76561198057612441 76561198065652166 76561198074532482 76561198079466268 76561198085552072 76561198086062224 76561198086567298 76561198087535915 76561198091618683 76561198124175171 76561198124353746 76561198141878688 76561198142431796 76561198180391006 76561198028688215 76561198042153111 76561198049098104 76561198055426325 76561198060377021 76561198067689729 76561198075881583 76561198087307481 76561198093434893 76561198117404218 76561198124502654 76561198145139833 76561198150324900 76561198152844530 76561198156506310 76561198172592282 76561198218941020 76561197974046961 76561197980844910 76561197984503593 76561197991562767 76561197992942411 76561198023084904 76561198034574769 76561198042625076 76561198044333695 76561198049950417 76561198053277219 
76561198053783902 76561198063542775 76561198063637267 76561198063905710 76561198065438271 76561198068747085 76561198071036862 76561198078805664 76561198082034292 76561198085434442 76561198085696758 76561198088588549 76561198091551547 76561198098787581 76561198099400043 76561198100804737 76561198101489666 76561198108502306 76561198109890199 76561198113490250 76561198115197700 76561198118095458 76561198118492542 76561198120939126 76561198121046738 76561198121607034 76561198124847087 76561198126005089 76561198126752265 76561198130651979 76561198136062360 76561198138972128 76561198144494256 76561198147112715 76561198149394684 76561198150135022 76561198150321509 76561198150617017 76561198153498776 76561198156949251 76561198170942770 76561198205970008 76561198207751988 76561198067201275

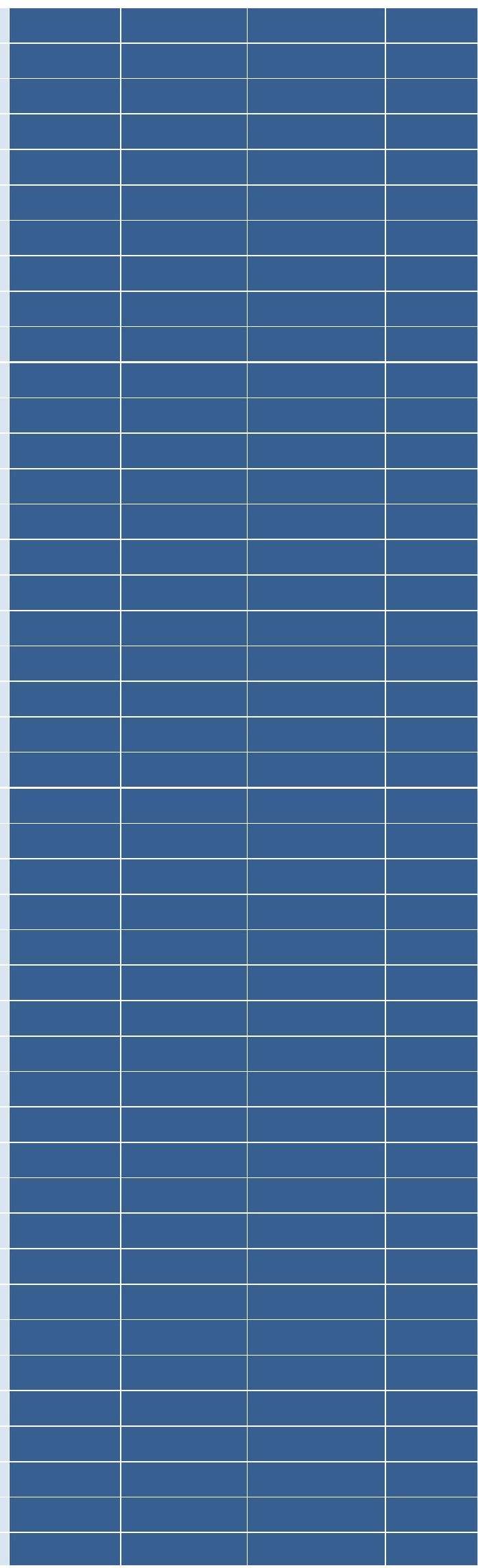




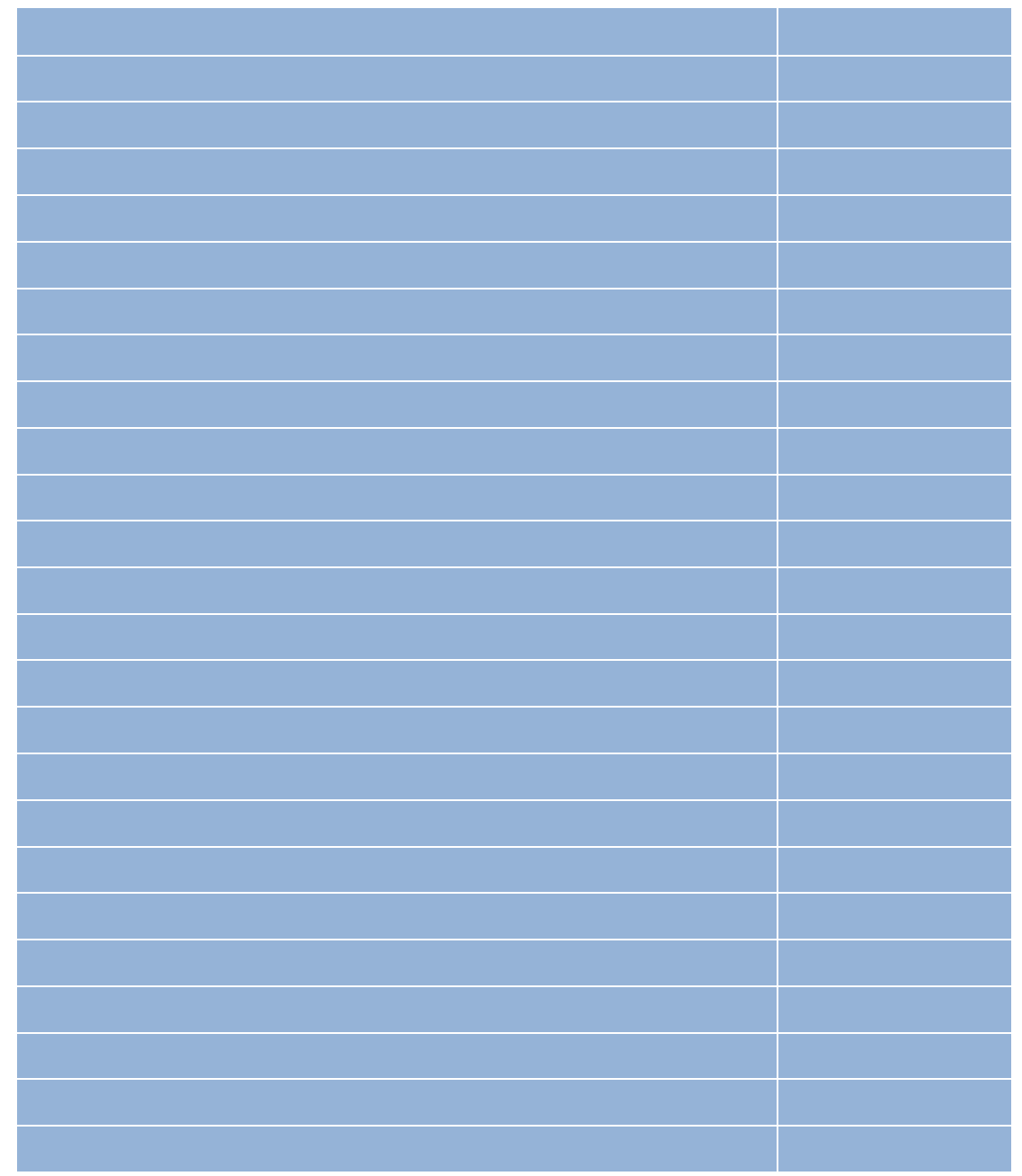

\begin{tabular}{|l|l|}
\hline Readability Metric & Value \\
\hline & \\
\hline & \\
\hline & \\
\hline
\end{tabular}

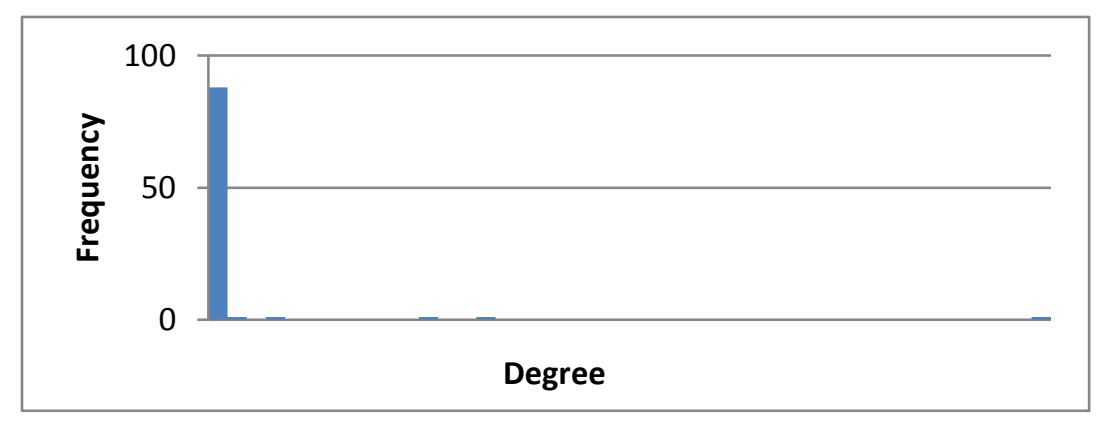

\begin{tabular}{|l|r|}
\hline Minimum Degree & 1 \\
\hline Maximum Degree & 55 \\
\hline Average Degree & 2.022 \\
\hline Median Degree & 1.000 \\
\hline
\end{tabular}




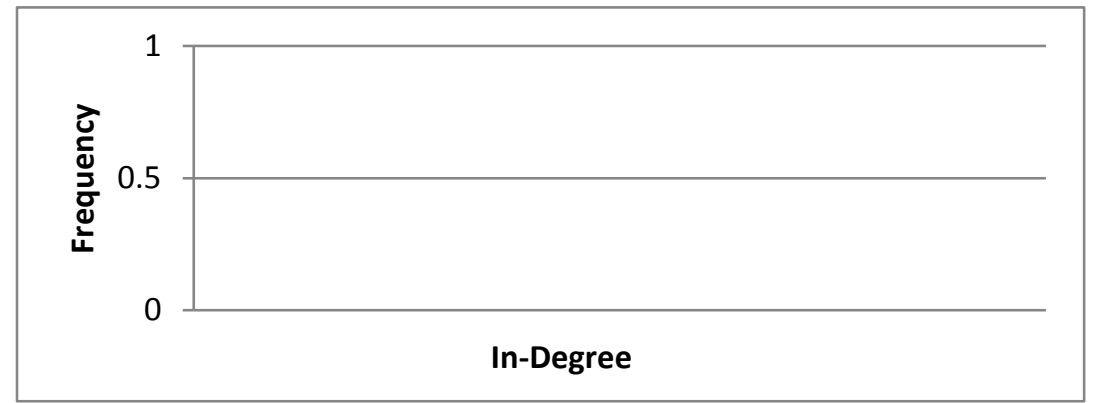

\begin{tabular}{|l|l|}
\hline Minimum In-Degree & Not Available \\
\hline Maximum In-Degree & Not Available \\
\hline Average In-Degree & Not Available \\
\hline Median In-Degree & Not Available \\
\hline
\end{tabular}

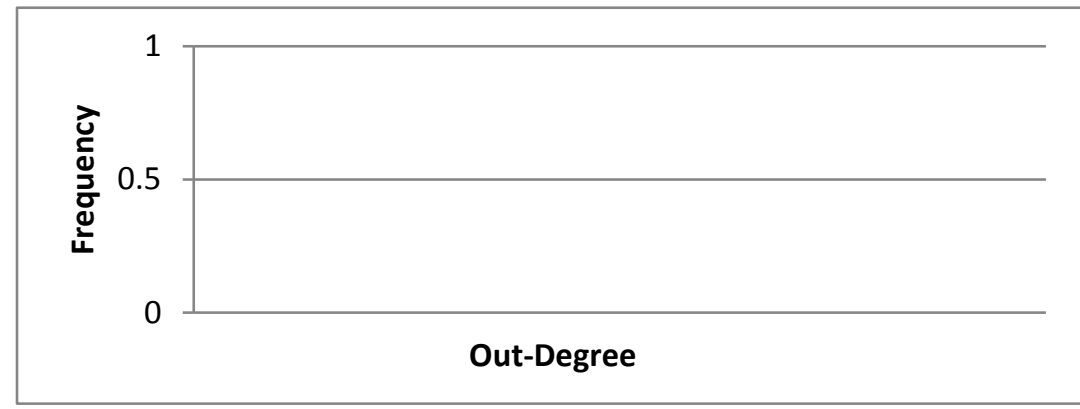

\begin{tabular}{|l|l|}
\hline Minimum Out-Degree & Not Available \\
\hline Maximum Out-Degree & Not Available \\
\hline Average Out-Degree & Not Available \\
\hline Median Out-Degree & Not Available \\
\hline
\end{tabular}

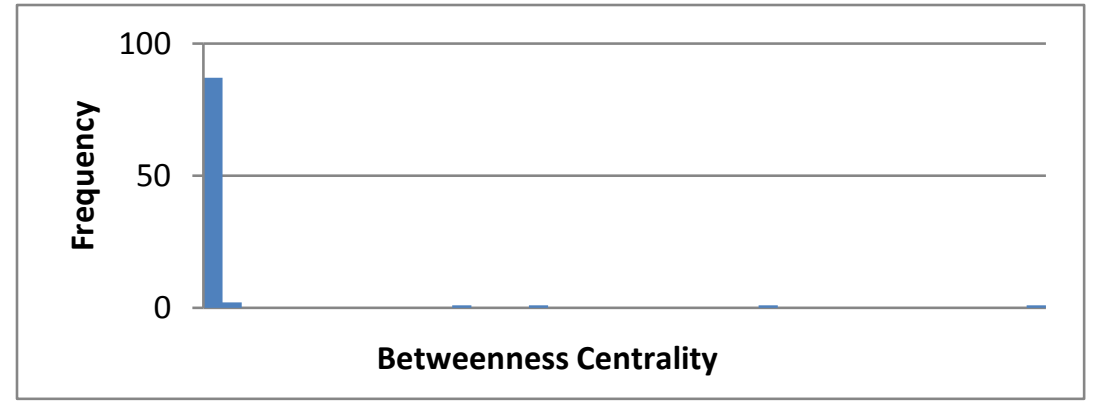

Minimum Betweenness Centrality

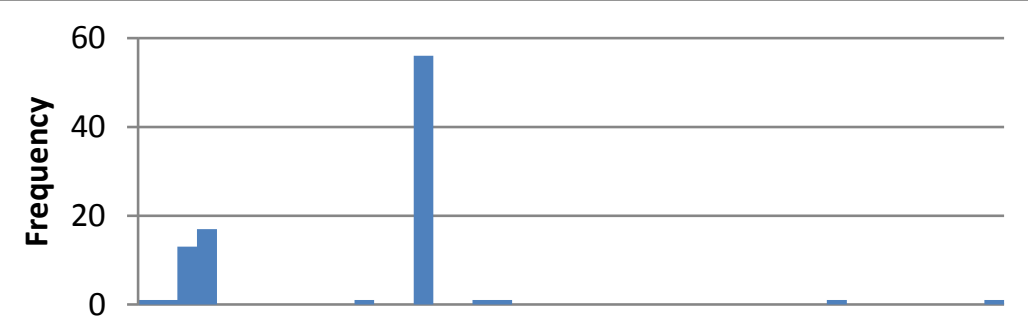




\begin{tabular}{|l|l|}
\hline Minimum Closeness Centrality & 0.003 \\
\hline Maximum Closeness Centrality & 0.006 \\
\hline Average Closeness Centrality & 0.004 \\
\hline Median Closeness Centrality & 0.004 \\
\hline
\end{tabular}

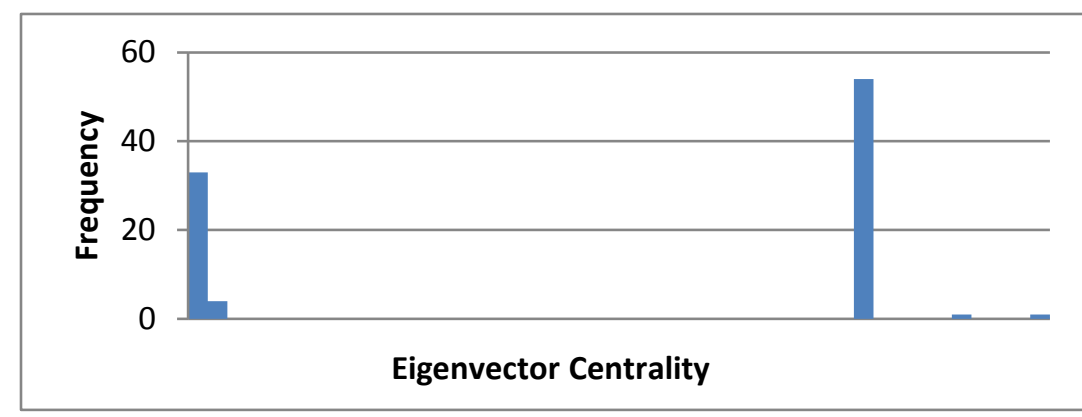

\begin{tabular}{|l|l|}
\hline Minimum Eigenvector Centrality & 0.000 \\
\hline Maximum Eigenvector Centrality & 0.021 \\
\hline Average Eigenvector Centrality & 0.011 \\
\hline Median Eigenvector Centrality & 0.017 \\
\hline
\end{tabular}

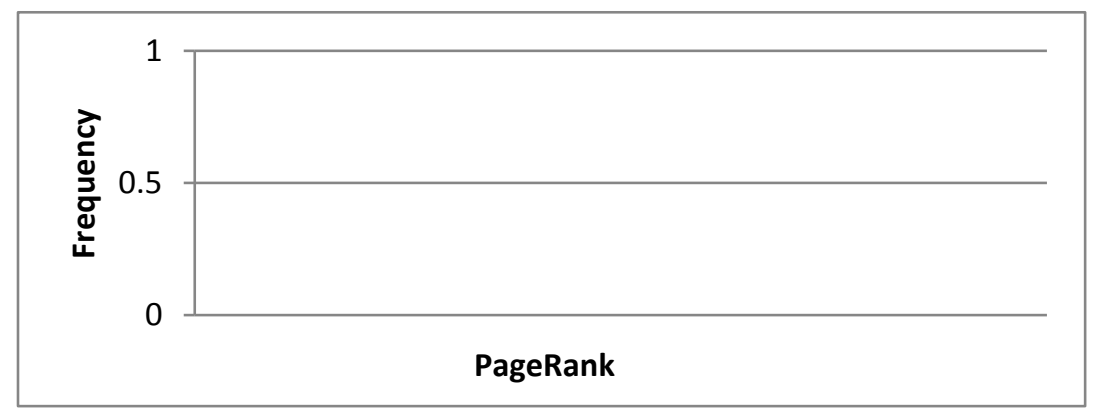

\begin{tabular}{|l|l|}
\hline Minimum PageRank & Not Available \\
\hline Maximum PageRank & Not Available \\
\hline Average PageRank & Not Available \\
\hline Median PageRank & Not Available \\
\hline
\end{tabular}

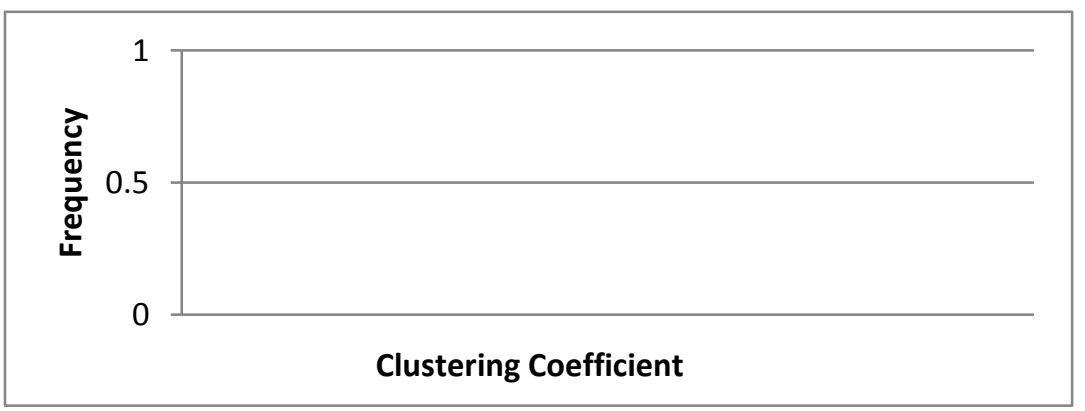




\begin{tabular}{|l|l|}
\hline Minimum Clustering Coefficient & Not Available \\
\hline Maximum Clustering Coefficient & Not Available \\
\hline Average Clustering Coefficient & Not Available \\
\hline Median Clustering Coefficient & Not Available \\
\hline
\end{tabular}

\begin{tabular}{|c|c|c|c|c|}
\hline Steamld & $\begin{array}{c}\text { Achievements } \\
\text { (TF) }\end{array}$ & Playerltems & $\begin{array}{l}\text { Time Played } \\
\text { (Min) }\end{array}$ & $\begin{array}{l}\text { Number of } \\
\text { Groups }\end{array}$ \\
\hline 76561198053783902 & profile not public & $\begin{array}{l}\text { permission } \\
\text { denied } \\
\text { permission }\end{array}$ & - & profile not public \\
\hline 76561198057612441 & profile not public & $\begin{array}{l}\text { denied } \\
\text { permission }\end{array}$ & - & profile not public \\
\hline 76561198098787581 & profile not public & $\begin{array}{l}\text { denied } \\
\text { permission }\end{array}$ & - & \\
\hline 76561198100804737 & profile not public & $\begin{array}{l}\text { denied } \\
\text { permission }\end{array}$ & - & \\
\hline 76561198120939126 & profile not public & $\begin{array}{l}\text { denied } \\
\text { permission }\end{array}$ & - & \\
\hline 76561198121607034 & profile not public & $\begin{array}{l}\text { denied } \\
\text { permission }\end{array}$ & - & \\
\hline 76561198124175171 & profile not public & $\begin{array}{l}\text { denied } \\
\text { permission }\end{array}$ & - & profile not public \\
\hline 76561198126752265 & profile not public & $\begin{array}{l}\text { denied } \\
\text { permission }\end{array}$ & - & \\
\hline 76561198147112715 & profile not public & $\begin{array}{l}\text { denied } \\
\text { permission }\end{array}$ & - & \\
\hline 76561198150321509 & profile not public & $\begin{array}{l}\text { denied } \\
\text { permission }\end{array}$ & - & \\
\hline 76561198121046738 & 412 & $\begin{array}{l}\text { denied } \\
\text { permission }\end{array}$ & 2706 & \\
\hline 76561198074532482 & 293 & $\begin{array}{l}\text { denied } \\
\text { permission }\end{array}$ & 38258 & 9 \\
\hline 76561198113490250 & 217 & $\begin{array}{l}\text { denied } \\
\text { permission }\end{array}$ & 71480 & \\
\hline 76561198126005089 & 202 & $\begin{array}{l}\text { denied } \\
\text { permission }\end{array}$ & 38511 & \\
\hline 76561197992942411 & 193 & $\begin{array}{l}\text { denied } \\
\text { permission }\end{array}$ & 10267 & 7 \\
\hline 76561198101489666 & 186 & $\begin{array}{l}\text { denied } \\
\text { permission }\end{array}$ & 12925 & \\
\hline 76561198152532873 & 113 & denied & 14024 & 10 \\
\hline 76561198170942770 & 97 & permission & 3565 & \\
\hline
\end{tabular}


76561198150135022

76561198142431796

76561198132684906

76561198218941020

76561198086062224

76561198082034292

76561198065652166

76561198149237454

76561198205970008

76561198067201275

76561198129085144

76561198086567298

76561198109890199

76561198207751988

76561198023084904

76561198087307481

76561198124847087

76561198156506310

76561198118492542

76561197984503593

76561198150617017

76561198153498776

76561198034574769 denied

permission

81 denied

permission

61 denied 4824

5

permission

39 denied

947

4

permission

32 denied

permission

25 denied

402

2

626

8

permission

20 denied 283

permission

19 denied

permission

19 denied

permission

18 denied

permission

17 denied 638

permission

14 denied 231

permission

13 denied 249

permission

13 denied 251

permission

13 denied 233

permission

10 denied 258

permission

10 denied 210

permission

10 denied 341

permission

7 denied 364

permission

5 denied 200

permission

3 denied

116

permission

3 denied 30

permission

3 denied 60

permission

1 denied
2

0

3

1

3

5

8 
76561198150324900

76561197991562767

76561198053277219

76561198055426325

76561198063637267

76561198063905710

76561198085434442

76561198085696758

76561198087535915

76561198099400043

76561198115197700

76561198124353746

76561198138972128

76561198141878688

76561198144494256

76561198148113785

76561198149394684

76561198180391006

76561198239562328

76561198093434893

76561198075881583

76561197974046961

76561198079466268

76561198071036862

76561198065438271

76561198117404218

76561198060377021

76561198136062360 permission

1 denied permission

0 denied permission

0 denied permission

0 denied permission

0 denied permission

0 denied permission

0 denied permission

0 denied 20 16 permission

0 denied permission

0 denied permission

0 denied permission

0 denied permission

0 denied permission

0 denied permission

0 denied permission

0 denied permission

0 denied permission

0 denied permission

0 denied

16

182

313

373

221

303

352

193

119

217
436

434

420

378

329

295

220

200

198
0

4

7

1

1

2

0

0

0

0

0

0

0

0

0

0

0

0

0

5

0 43

32

120

9

116

12

15 
76561198091551547

76561198091618683

76561198068747085

76561198078805664

76561198049098104

76561198044333695

76561197980844910

76561198156949251

76561198042153111

76561198049950417

76561198028688215

76561198149180078

76561198118095458

76561198042625076

76561198085552072

76561198088588549

76561198108502306

76561198124502654

76561198067689729

76561198063542775

76561198130651979

76561198145139833

76561198152844530

76561198172592282
170

50

294

213

221

368

275

136

198

197

36

64

518

44

13

21

13

8

5

1

2

0

0

0
168

144

136

118

117

106

102

95

89

73

54

45

41

41

24

17

9

7

4

3

1

0

0

0
11224

2047

31795

11154

7877

12473

4829

2132

6899

10913

1631

6256

2036

1707

725

881

602

75

26

14

103

0

0

0
12

8

84

54

12

17

22

4

10

3

6

4

36

1

4

2
76561198149180078 76561198149180078 76561198149180078 76561198149180078 76561198149180078 76561198149180078 76561198129085144 76561198129085144 76561198132684906 76561198132684906 76561198132684906 76561198132684906 76561198132684906 76561198132684906 76561198132684906 76561198132684906 76561198132684906 76561198132684906
76561198129085144 76561198132684906 76561198148113785 76561198149237454 76561198152532873 76561198239562328 76561198149180078 76561198149237454 76561198057612441 76561198065652166 76561198074532482 76561198079466268 76561198085552072 76561198086062224 76561198086567298 76561198087535915 76561198091618683 76561198124175171 


\begin{tabular}{|c|c|}
\hline 76561198132684906 & 76561198124353746 \\
\hline 76561198132684906 & 76561198141878688 \\
\hline 76561198132684906 & 76561198142431796 \\
\hline 76561198132684906 & 76561198149180078 \\
\hline 76561198132684906 & 76561198239562328 \\
\hline 76561198148113785 & 76561198149180078 \\
\hline 76561198148113785 & 76561198180391006 \\
\hline 76561198149237454 & 76561198028688215 \\
\hline 76561198149237454 & 76561198042153111 \\
\hline 76561198149237454 & 76561198049098104 \\
\hline 76561198149237454 & 76561198055426325 \\
\hline 76561198149237454 & 76561198060377021 \\
\hline 76561198149237454 & 76561198067689729 \\
\hline 76561198149237454 & 76561198075881583 \\
\hline 76561198149237454 & 76561198087307481 \\
\hline 76561198149237454 & 76561198093434893 \\
\hline 76561198149237454 & 76561198117404218 \\
\hline 76561198149237454 & 76561198124502654 \\
\hline 76561198149237454 & 76561198129085144 \\
\hline 76561198149237454 & 76561198145139833 \\
\hline 76561198149237454 & 76561198149180078 \\
\hline 76561198149237454 & 76561198150324900 \\
\hline 76561198149237454 & 76561198152844530 \\
\hline 76561198149237454 & 76561198156506310 \\
\hline 76561198149237454 & 76561198172592282 \\
\hline 76561198149237454 & 76561198218941020 \\
\hline 76561198152532873 & 76561197974046961 \\
\hline 76561198152532873 & 76561197980844910 \\
\hline 76561198152532873 & 76561197984503593 \\
\hline 76561198152532873 & 76561197991562767 \\
\hline 76561198152532873 & 76561197992942411 \\
\hline 76561198152532873 & 76561198023084904 \\
\hline 76561198152532873 & 76561198034574769 \\
\hline 76561198152532873 & 76561198042625076 \\
\hline 76561198152532873 & 76561198044333695 \\
\hline 76561198152532873 & 76561198049950417 \\
\hline 76561198152532873 & 76561198053277219 \\
\hline 76561198152532873 & 76561198053783902 \\
\hline 76561198152532873 & 76561198063542775 \\
\hline 76561198152532873 & 76561198063637267 \\
\hline 76561198152532873 & 76561198063905710 \\
\hline 76561198152532873 & 76561198065438271 \\
\hline 76561198152532873 & 76561198068747085 \\
\hline 76561198152532873 & 76561198071036862 \\
\hline
\end{tabular}




\begin{tabular}{|c|c|}
\hline Connections & \\
\hline 76561198152532873 & 76561198078805664 \\
\hline 76561198152532873 & 76561198082034292 \\
\hline 76561198152532873 & 76561198085434442 \\
\hline 76561198152532873 & 76561198085696758 \\
\hline 76561198152532873 & 76561198088588549 \\
\hline 76561198152532873 & 76561198091551547 \\
\hline 76561198152532873 & 76561198098787581 \\
\hline 76561198152532873 & 76561198099400043 \\
\hline 76561198152532873 & 76561198100804737 \\
\hline 76561198152532873 & 76561198101489666 \\
\hline 76561198152532873 & 76561198108502306 \\
\hline 76561198152532873 & 76561198109890199 \\
\hline 76561198152532873 & 76561198113490250 \\
\hline 76561198152532873 & 76561198115197700 \\
\hline 76561198152532873 & 76561198118095458 \\
\hline 76561198152532873 & 76561198118492542 \\
\hline 76561198152532873 & 76561198120939126 \\
\hline 76561198152532873 & 76561198121046738 \\
\hline 76561198152532873 & 76561198121607034 \\
\hline 76561198152532873 & 76561198124847087 \\
\hline 76561198152532873 & 76561198126005089 \\
\hline 76561198152532873 & 76561198126752265 \\
\hline 76561198152532873 & 76561198130651979 \\
\hline 76561198152532873 & 76561198136062360 \\
\hline 76561198152532873 & 76561198138972128 \\
\hline 76561198152532873 & 76561198144494256 \\
\hline 76561198152532873 & 76561198147112715 \\
\hline 76561198152532873 & 76561198149180078 \\
\hline 76561198152532873 & 76561198149394684 \\
\hline 76561198152532873 & 76561198150135022 \\
\hline 76561198152532873 & 76561198150321509 \\
\hline 76561198152532873 & 76561198150617017 \\
\hline 76561198152532873 & 76561198153498776 \\
\hline 76561198152532873 & 76561198156949251 \\
\hline 76561198152532873 & 76561198170942770 \\
\hline 76561198152532873 & 76561198205970008 \\
\hline 76561198152532873 & 76561198207751988 \\
\hline 76561198239562328 & 76561198067201275 \\
\hline 76561198239562328 & 76561198132684906 \\
\hline 76561198239562328 & 76561198149180078 \\
\hline
\end{tabular}


A Qualitative Analysis of a Team Fortress 2 Social Network 
146796||

Social capital, self-esteem, and use of online social network sites: A longitudinal analysis

- Charles Steinfield,

- Nicole B. Ellison,

- Cliff Lampe

- Department of Telecommunication, Information Studies, and Media, Michigan State University, East Lansing, MI 48824 USA Available online 17 August 2008

14 Lazarsfeld, Berelson and Gaudet, op. cit., pp. 49-50 . http://www.gamesradar.com/history-of-valve/, 2013),

Recommended Citation Katz, E. (1957). The Two-Step Flow of Communication: An Up-To-Date Report on an Hypothesis. Political Opinion Quarterly, 21 (1), 61 78. http://dx.doi.org/10.1086/266687

https://en.wikipedia.org/wiki/The_Tipping_Point https://en.wikipedia.org/wiki/Embeddedness 
Electronic Commerce Research and Applications, Volume 13, Issue 6, November-December 2014, Pages 431-439

Yen-Liang Chen, Kwei Tang, Chia-Chi Wu, Ru-Yun Jheng 